Pecvnia, 9 (2009), pp. 205-223

\title{
Análisis del grado de sensibilización del empresario ante el problema del empleo de trabajadores con discapacidad en la provincia de Cádiz
}

Recibido: Diciembre 2009

Aceptado: Marzo 2010

\author{
Héctor M. Ramos Romero \\ hector.ramos@uca.es \\ Universidad de Cádiz \\ Fac. de Ciencias Económicas y Empresariales \\ Duque de Nájera, 8 \\ 11002 Cádiz (España) \\ Antonio Leal Jiménez \\ antonio.leal@uca.es \\ Universidad de Cádiz \\ Fac. de Ciencias Sociales y de la Comunicación \\ Av. de la Universidad, $\mathrm{s} / \mathrm{h}$ \\ 11405 Jerez de la Fra. (Cádiz, España)
}

Proponemos en este trabajo un indicador para evaluar el grado de sensibilización del empresario ante el problema de la contratación de trabajadores con discapacidad en la provincia de Cádiz. A partir de los datos obtenidos a través de entrevistas personales a empresarios, llevamos a cabo un análisis de la varianza multifactorial y estudiamos la influencia sobre el indicador de factores como son el sector de actividad de la empresa, la existencia previa de trabajadores con
This paper presents an indicator to evaluate employer's attitudes towards the employment of disabled people in the Province of Cadiz.

Using data collected during personal interviews with employers, we applied a multifactor ANOVA test to analyse which factors influence the indicator values. The factors considered are the sector of business activity, the company size, the prior existence of disabled 
discapacidad y el tamaño de la empresa, así como sus interacciones.

Palabras clave: trabajadores con discapacidad, contratación laboral, sensibilización empresarial. workers in the company, and their interactions.

Key words: employment of disabled people, recruitment, disability awareness.

\section{INTRODUCCIÓN}

En 1982 se promulgó en nuestro país la Ley 13/1982 de 7 de abril, de Integración Social de los Minusválidos (LISMI) que desarrolla el artículo 49 de nuestra Constitución Española en el que se proclama la dignidad propia de las personas con discapacidad para su completa realización personal y su total integración social. Desde la aparición de la ley se ha venido avanzando hasta completarse un importante marco legal.

Los empresarios, en general, relacionan directamente su política de empleo con el éxito de sus negocios. Es evidente que a la hora de contratar a personas con discapacidad siguen también esta filosofía. Además, ciertas actitudes de los empresarios y los prejuicios de un entorno que no favorece plenamente sus necesidades, provocan que las personas con discapacidad tengan verdaderos problemas a la hora de acceder al mercado laboral. Asimismo, encuentran dificultades en la adquisición de una formación suficiente que les permita desarrollar trabajos adecuados.

El trabajo que aquí se presenta surge a partir de los datos obtenidos en un amplio estudio financiado por el Ministerio de Trabajo y Asuntos Sociales que desarrollamos durante el año 2006 a través de una investigación de campo en empresas de la provincia de Cádiz. Ese estudio, fruto de un convenio de la Universidad de Cádiz con el IMSERSO a través del Centro de Recuperación de Minusválidos Físicos (CRMF) de S. Fernando (Cádiz), tenía como objetivo el analizar las oportunidades en el empleo para personas con discapacidad física y sensorial en la provincia de Cádiz. En el mismo, dentro de un marco de investigación esencialmente cualitativa, se valora la actitud y la opinión de expertos y la de los empresarios, con el objetivo de diagnosticar cuál es la actitud de las empresas gaditanas en relación con su política de contratación de personas con discapacidad y, de esa manera, disponer de un marco de referencia para futuras actuaciones dirigidas a apoyar la integración laboral del colectivo de personas con discapacidad. El estudio se lleva a cabo mediante un cuestionario en el que se abordan cuestiones relativas a diferentes aspectos como son: nivel 
de conciencia, nivel de conocimiento, nivel de contratación y conservación de empleo $y$, finalmente, nivel de imagen.

En el trabajo que aquí se presenta estamos interesados en particular en el análisis del grado de sensibilidad del empresario ante el empleo de trabajadores con discapacidad. Para abordar esta cuestión nos centraremos únicamente en las respuestas a cuatro ítems relativos a nivel de contratación y conservación de empleo y en un ítem relativo a nivel de imagen. Las diferentes categorías de respuestas dadas a estos cinco ítems nos permitirá obtener un indicador sintético del grado de sensibilidad del empresario como empleador potencial de trabajadores con discapacidad. Una vez obtenido el indicador llevaremos a cabo un análisis de la influencia de determinados factores sobre el indicador de sensibilización.

\section{METODOLOGÍA}

\subsection{El cuestionario}

Para adquirir una aproximación cualificada de las particularidades del escenario en el que se desarrollaría el trabajo de investigación, se decidió hacer una serie de entrevistas a expertos pertenecientes a diferentes organismos que trabajan de una manera $u$ otra en el ámbito laboral y/o ámbito de la discapacidad y a directivos de empresas representativas en relación con este tema. Concretamente, como expertos participaron:

- Confederación de Empresarios de Cádiz

- Asociación de Jóvenes Empresarios

- Instituto de Fomento y Empleo del Ayuntamiento de Cádiz

- Unidad Territorial de Empleo, Desarrollo Local y Tecnológico

- Centro de Valoración y Orientación de la Junta de Andalucía

- Federación Gaditana de Personas con Discapacidad Física

- Directivos de empresas de menos de 50 trabajadores

- Directivos de empresas de más de 50 trabajadores

A partir de esa fase previa se elabora un cuestionario con un total de 21 preguntas agrupadas en cuatro apartados: nivel de conciencia, nivel de conocimiento, nivel de contratación y conservación de empleo y, finalmente, nivel de imagen. 
Para el desarrollo del presente trabajo solo vamos a estar interesados en las respuestas a los siguientes ítems:

1) En los procesos de contratación en los que también hay personas con discapacidad, ¿piensa usted que se tiene en cuenta el valor de la igualdad de oportunidades y en general de las buenas prácticas de contratación?

2) ¿En qué sentido considera que el ambiente laboral puede verse afectado al incluirse a una persona con discapacidad en la plantilla?

3) ¿Considera usted que la posibilidad de un mayor índice de "absentismo" es un handicap a la hora de contratar a una persona con discapacidad?

4) A igualdad de condiciones y suponiendo que ha contratado, ipercibe diferencias en el rendimiento entre personas con discapacidad y el resto?

5) ¿Considera que la contratación de personas con discapacidad puede influir en la imagen de la empresa? ¿Cómo?

\subsection{La muestra}

En primer lugar, para garantizar que la muestra incluyera empresas con empleados con discapacidad, se consideró una estratificación basada en el tamaño de la empresa (menos de 50 trabajadores y más de 50 trabajadores), dada la obligación legal de contratar este tipo de trabajadores -al margen de otras medidas subsidiarias o alternativas- en el caso de las empresas con más de 50 trabajadores.

Tamaño de la muestra realizada: 60 entrevistas:

- 30 empresas con más de 50 trabajadores

- 30 empresas con menos de 50 trabajadores

Puesto que a priori la percepción desde las empresas del problema de las oportunidades en el empleo de personas de estas características puede depender de que la empresa tenga la experiencia o no de contar con personas con discapacidad entre sus empleados, nos pareció conveniente garantizar en la muestra un número representativo de empresas que tuvieran empleados de dicho grupo. En este sentido, utilizando diversas fuentes de información, incluimos en la muestra 
empresas con empleados con discapacidad. Todo ello de forma predeterminada y representando un porcentaje ligeramente superior al $50 \%$ de la muestra (concretamente, 32 empresas sobre el total de 60 ). El resto de la muestra fue seleccionado aleatoriamente. Por otra parte, se buscó que el ámbito geográfico de las tres Cámaras de Comercio existentes en la provincia de Cádiz estuviera representado en la muestra de empresas encuestadas.

Por sectores, la descripción de la muestra es la siguiente:

- Sector servicios: 42 empresas

- Sector industrial: 15

- Administración pública: 3

\section{RESULTADOS}

\subsection{Resultados descriptivos}

3.1.1. Características de las empresas encuestadas

De las 60 empresas estudiadas constatamos que 36 de ellas tenían contratados a trabajadores con discapacidad (Figura 1).

De las 24 empresas que no tenían contratadas a ninguna persona con discapacidad observamos que 5 empresas habían tenido al menos alguna experiencia laboral con dichas personas, ya sea por contrato o por prácticas de empresa. Por lo tanto, en nuestra muestra tenemos 19 empresas (32\%) que no habían tenido ninguna experiencia laboral con personas discapacitadas.

En la muestra encontramos 7 empresas, de 50 ó más trabajadores, que no habían tenido ninguna experiencia laboral, ni siquiera de prácticas, con personas discapacitadas, a pesar de que el Art. 38 de la Ley 13/1982, de 7 de abril, de Integración Social de los Minusválidos, exige la contratación de un número de trabajadores discapacitados no inferior del $2 \%$ de la plantilla, para empresas públicas o privadas que empleen a un número de trabajadores fijos que exceda de cincuenta. 
Figura 1

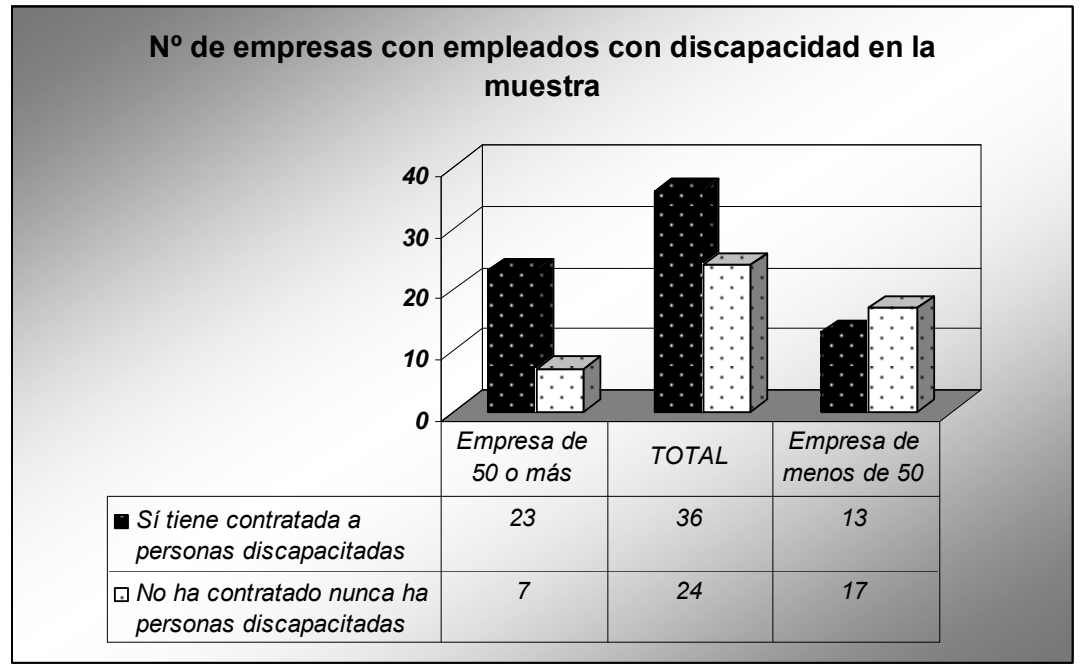

En cuanto a la posibilidad de optar por medidas alternativas al cumplimiento de la reserva del 2\% (Real Decreto 364/2005, de 8 de abril, por el que se regula el cumplimiento alternativo con carácter excepcional de la cuota de reserva a favor de los trabajadores con discapacidad) comprobamos que sólo una empresa de estas 7 afirmó que colaboraba con centros especiales de empleo.

Figura 2

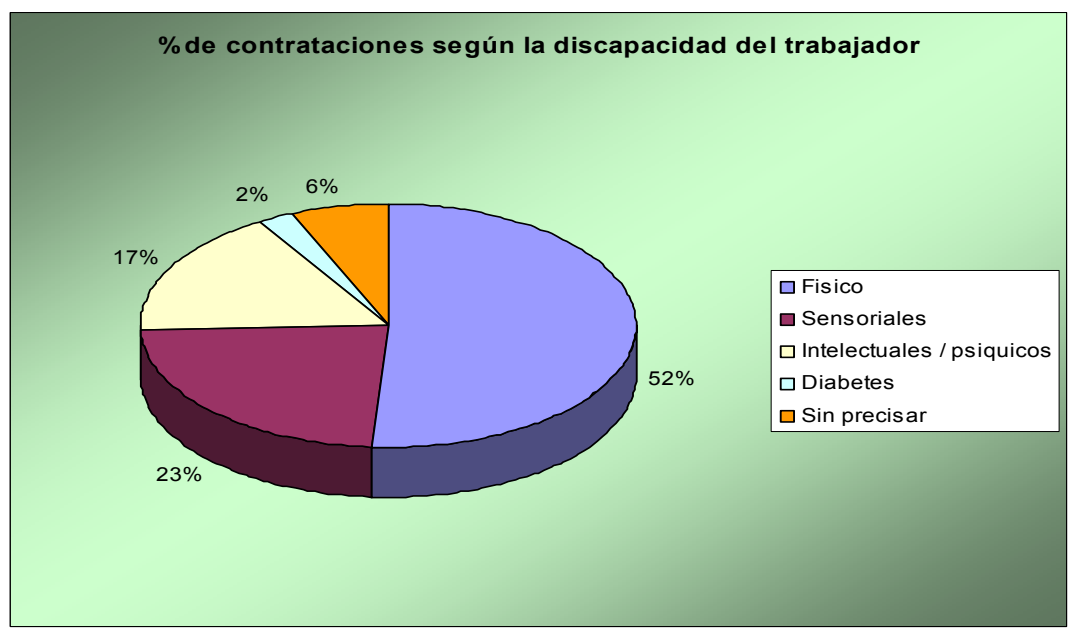


Por otra parte, pudimos observar que, en las empresas donde se tenía experiencia con trabajadores con discapacidad (Figura 2), el 52\% de los casos se trataba de personas con discapacidad física, seguido por contrataciones de trabajadores con discapacidad sensorial (23\%) e intelectual o psíquica (17\%).

\subsubsection{Nivel de contratación y conservación de empleo}

\section{Item 1:}

En los procesos de contratación en los que también hay personas con discapacidad, ¿piensa usted que se tiene en cuenta el valor de la igualdad de oportunidades y en general de las buenas prácticas de contratación?

La opinión de las empresas estaba muy dividida en relación a esta cuestión. Para algunas empresas la igualdad estaba apoyada en las ventajas fiscales por la contratación de trabajadores con discapacidad. Las empresas que consideraban que había discriminación defendían la idea de que para las empresas era mejor un trabajador que no tuviera discapacidad. Las empresas que opinaban que había igualdad creían en el trabajador porque simplemente buscaban al candidato más cualificado y que mejor pudiera desarrollar su puesto.

Por otra parte es de destacar la opinión mayoritaria acerca de que en la contratación pública era donde existía menos discriminación a la hora de contratar.

No se observó grandes diferencias en este tema entre empresas grandes y pequeñas, si bien la opinión de existencia de igualdad era mayor en el caso de las empresas de más de 50 trabajadores.

En el siguiente cuadro (Tabla 1) se recoge la opinión de las empresas al respecto, distinguiendo entre empresas con al menos 50 trabajadores y empresas con menos de 50 empleados, así como la opinión global.

Tabla 1

\begin{tabular}{|l|c|c|}
\cline { 2 - 3 } \multicolumn{1}{c|}{} & Hay discriminación & Hay igualdad \\
\hline 50 trabajadores o más & $47 \%$ & $53 \%$ \\
\hline Menos de 50 trabajadores & $59 \%$ & $41 \%$ \\
\hline Global & $53 \%$ & $47 \%$ \\
\hline
\end{tabular}




\section{Item 2:}

¿En qué sentido considera que el ambiente laboral puede verse afectado al incluirse a una persona con discapacidad en la plantilla?

En general se opinaba que la influencia de incluir en la plantilla a un trabajador con discapacidad iba a depender fundamentalmente de si la contratación había sido justa y el trabajador con discapacidad demostraba su valía. Es de destacar que la opinión de que la influencia era negativa era sensiblemente mayor en el caso de las empresas con más de 50 trabajadores que en las pequeñas empresas (véase Figura 3).

\section{Figura 3}

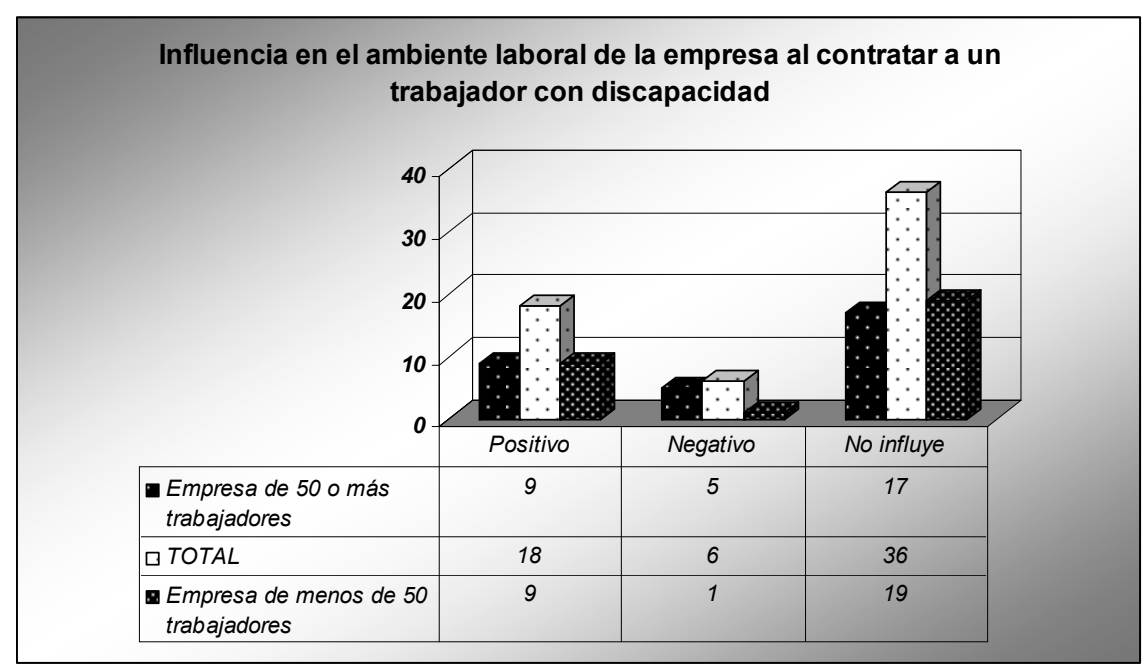

Item 3:

¿Considera usted que la posibilidad de un mayor índice de "absentismo" es un handicap a la hora de contratar a una persona con discapacidad?

El $60 \%$ de las empresas no consideraban que fuera un handicap el posible mayor índice de absentismo que pudiesen tener los trabajadores con discapacidad. Opinaban que el absentismo dependía más 
de la persona que de la discapacidad, aunque se reconocía que en los casos de gran discapacidad sí podía ser importante el grado de absentismo.

Es de destacar que la opinión más desfavorable se manifestaba en mayor grado entre las empresas con menos de 50 trabajadores: el 52\% consideraba el absentismo de personas con discapacidad como un handicap para ocupar un puesto de trabajo (Fig.4).

Figura 4

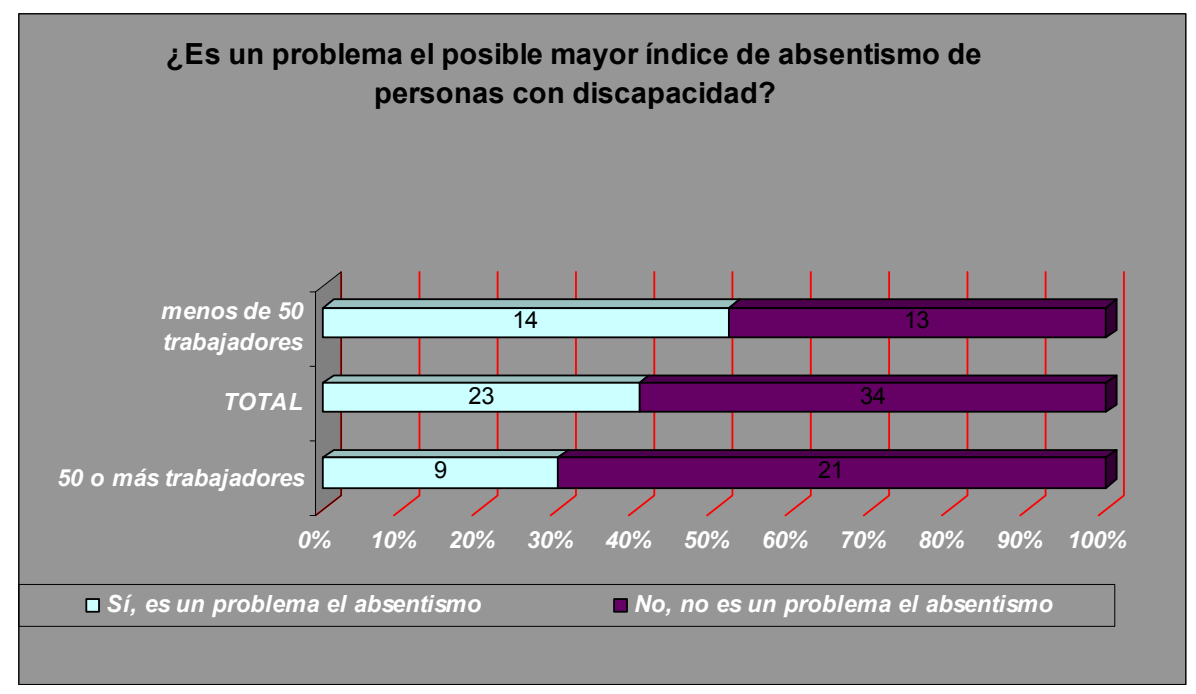

Item 4:

A igualdad de condiciones y suponiendo que ha contratado, ¿percibe diferencias en el rendimiento entre personas con discapacidad y el resto?

El $60 \%$ de las empresas consideraba que no se percibían diferencias entre un trabajador con discapacidad y otro trabajador que no tuviera discapacidad. En función del tamaño de la empresa se observó sensibles diferencias (véase Tabla 2). En particular, en las empresas con menos de 50 trabajadores era muy grande el porcentaje de las que no opinaban al respecto, probablemente por la falta de experiencia con este colectivo de trabajadores.

Se volvía a repetir la idea de que dependía más de la persona que de la propia discapacidad, su dedicación y adecuación al puesto. A lo 
largo de las entrevistas se constataba la opinión generalizada de que los trabajadores con discapacidad solían esforzarse más.

Tabla 2

\begin{tabular}{|l|c|c|c|}
\cline { 2 - 4 } \multicolumn{1}{c|}{} & Existen diferencias & No hay diferencias & NS/NC \\
\hline M0 trabajadores o más & $20 \%$ & $77 \%$ & $3 \%$ \\
\hline Genos de 50 trabajadores & $44 \%$ & $43 \%$ & $13 \%$ \\
\hline
\end{tabular}

\subsubsection{Nivel de imagen}

Item 5:

¿Considera que la contratación de personas con discapacidad puede influir en la imagen de la empresa? ¿Cómo?

El $77 \%$ de las empresas encuestadas opinaba que la imagen de la empresa se veía influenciada -positiva o negativamente- al tener personas contratadas con discapacidad. Mayoritariamente, la influencia era positiva aunque había un $5 \%$ de las empresas que considera que su imagen se vería influenciada de manera positiva o negativa dependiendo de la actitud de los clientes frente a las discapacidades.

En función del tamaño, en el caso de empresas de más de 50 trabajadores observamos que el $70 \%$ de ellas consideraba que influía en su imagen la contratación de trabajadores con discapacidad. La influencia que se observaba en este tipo de empresas era mayoritariamente positiva, aunque existía un $9 \%$ que consideraba que dependía de la actitud de clientes y proveedores.

En las empresas de menos de 50 trabajadores se observó que aumentaba al $83 \%$ el porcentaje de empresas que consideraban que su imagen se veía influenciada mediante la contratación de trabajadores con discapacidad (Tabla 3).

Tabla 3

\begin{tabular}{|l|c|c|}
\cline { 2 - 3 } \multicolumn{1}{c|}{} & Influye en la imagen & No influye \\
\hline 50 trabajadores o más & $70 \%$ & $30 \%$ \\
\hline Menos de 50 trabajadores & $83 \%$ & $17 \%$ \\
\hline Global & $77 \%$ & $23 \%$ \\
\hline
\end{tabular}


En el colectivo de empresas que consideran que la influencia es positiva, podemos distinguir (Figura 5):

a) Aquellas que creían en la igualdad de todas las personas a pesar de sus diferencias $(47 \%)$

b) Las que consideraban que este tipo de contrataciones les podía beneficiar mediante una mejor imagen de cara a sus clientes y proveedores $(25 \%)$

c) Las que consideran que tenían un compromiso social de cara a la sociedad ayudando a la eliminación de barreras psicológicas con respecto a las personas con discapacidad (14\%)

d) Las que opinaban que ello les beneficiaba en concursos públicos por tener este tipo de trabajadores (9\%)

Figura 5

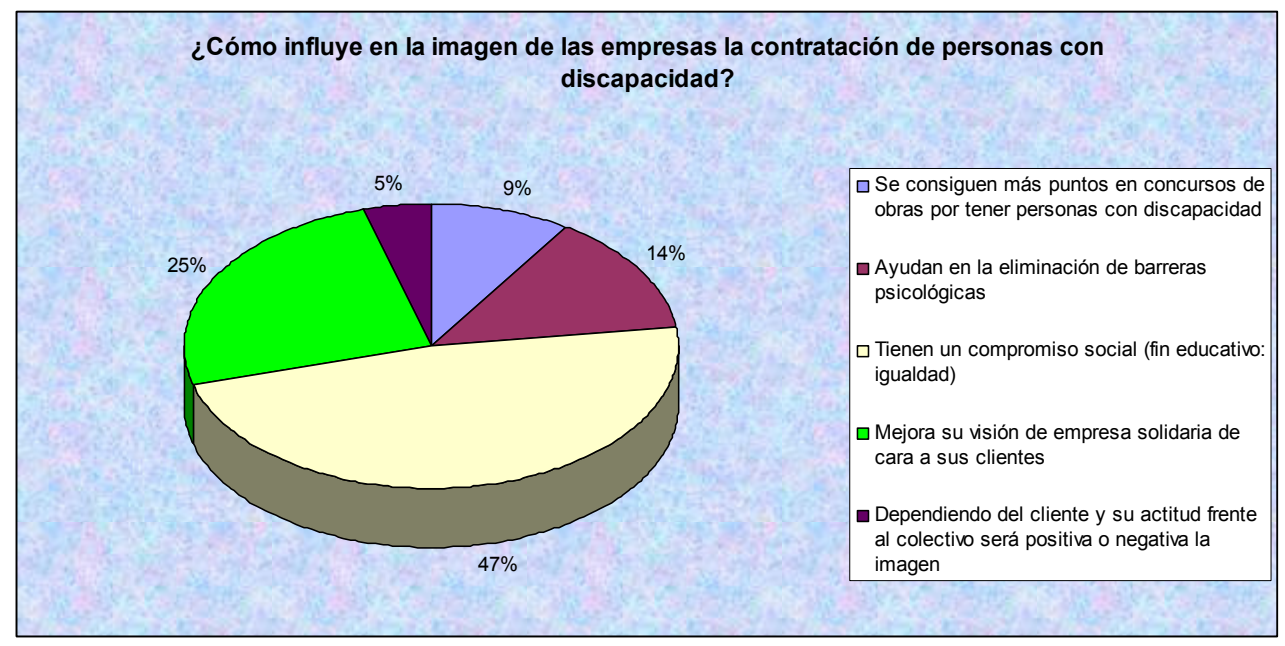

Por otra parte, en las Figuras 6 y 7, respectivamente, aparecen recogidos los correspondientes porcentajes cuando consideramos separadamente las empresas en función del número de trabajadores. 
Figura 6

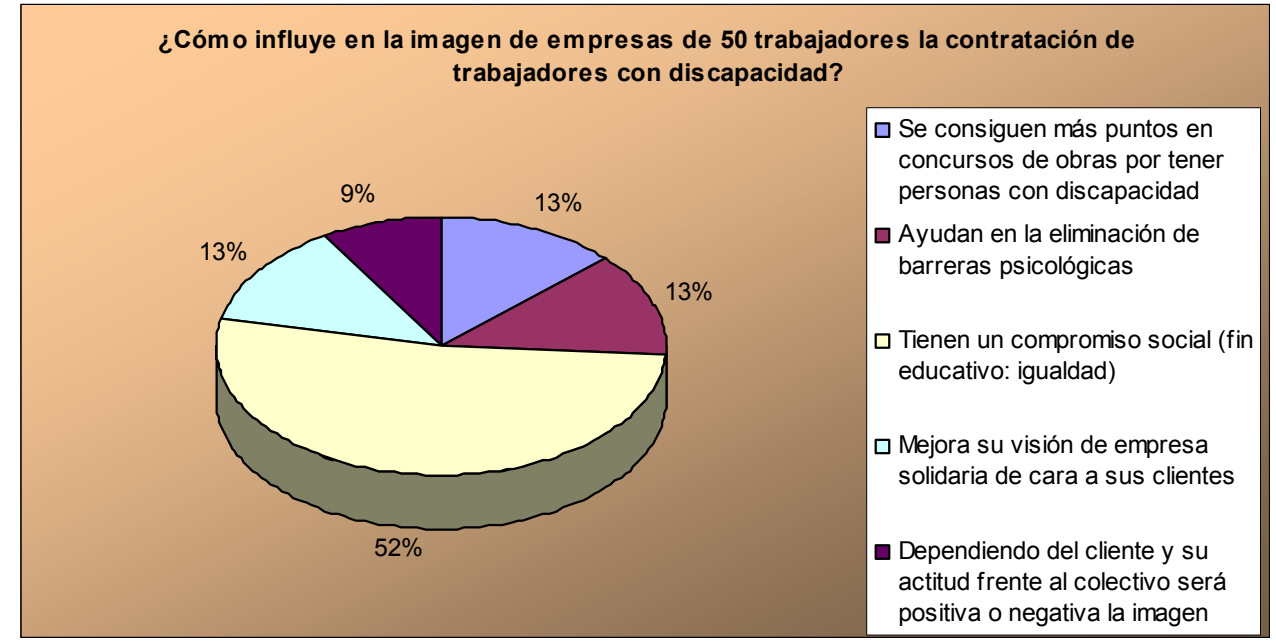

Figura 7

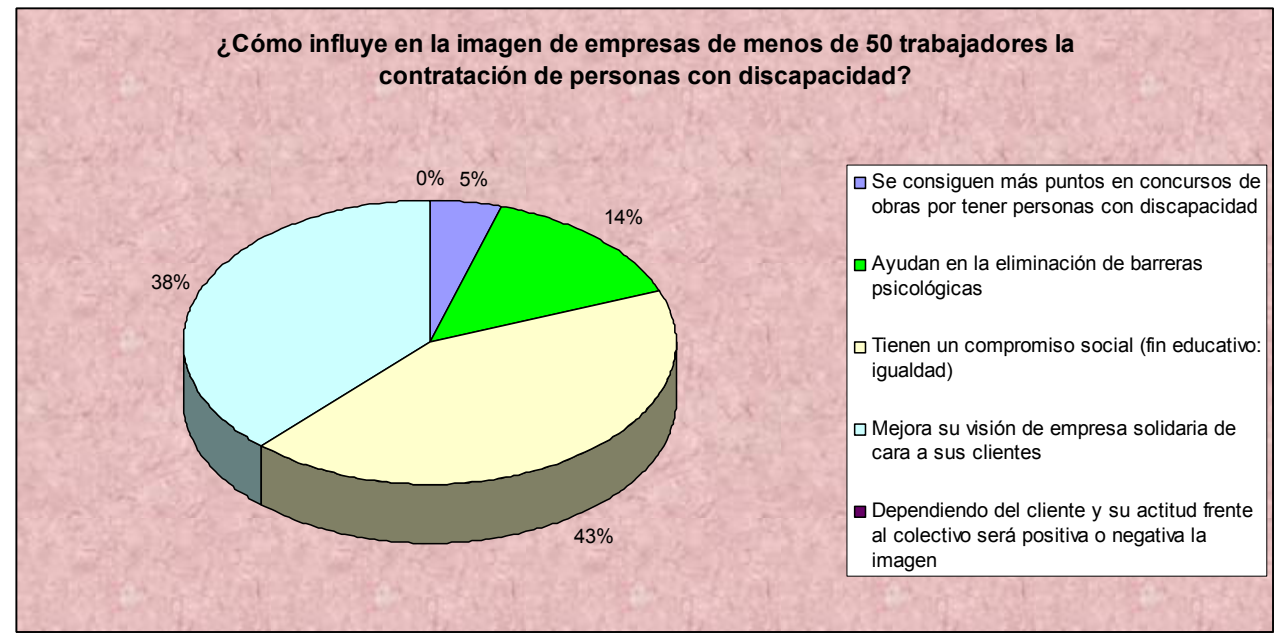

3.2. Análisis del grado de sensibilización del empresario ante el problema del empleo de trabajadores con discapacidad

Las diferentes categorías de respuestas dadas a estas cinco preguntas nos permiten obtener un indicador sintético de sensibilización del empresario ante el problema del empleo de trabajadores con discapacidad. Para ello las respuestas han sido clasificadas en positivas, negativas o neutras con puntuaciones respectivas de $+1,-1$ y 0 . El cómputo de las puntuaciones en esos apartados da lugar a un valor entero 
que se moverá en el rango teórico $[-5,5]$, si bien una vez evaluado se han obtenido valores enteros dentro del rango $[-2,5]$. Cuanto mayor sea el valor del indicador obtenido mayor es el grado de sensibilidad mostrado ante el problema en estudio.

Vamos a considerar los factores:

1) Sector (Servicios, Industrial, Administración pública)

2) Existencia de trabajadores con discapacidad (Sí / No)

3) Tamaño de la empresa (Más de 50 trabajadores, Menos de 50)

y vamos a analizar la influencia de estos factores sobre el indicador de sensibilización. Para ello llevaremos a cabo un análisis de la varianza multifactorial.

Obtenemos la siguiente tabla de resultados:

Tabla 4

\begin{tabular}{|l|c|c|c|c|c|}
\hline Fuente & $\begin{array}{c}\text { Suma de } \\
\text { cuadrados }\end{array}$ & $\begin{array}{c}\text { Grados } \\
\text { libertad }\end{array}$ & $\begin{array}{c}\text { Cuadrado } \\
\text { medio }\end{array}$ & Cociente F & p-valor \\
\hline Efectos principales & & & & & \\
\hline Sector & 17,1881 & 2 & 8,59405 & 2,30 & 0,1101 \\
\hline Tamaño & 12,6413 & 1 & 12,6413 & 3,38 & 0,0714 \\
\hline Trab. con discapacidad & 22,8261 & 1 & 22,8261 & 6,10 & 0,0166 \\
\hline RESIDUOS & 205,771 & 55 & 3,74129 & & \\
\hline TOTAL (Corregido) & 254,583 & 59 & & & \\
\hline
\end{tabular}

Podemos ver, de entrada, que en cierto sentido los tres factores considerados influyen en la variable respuesta ya que las correspondientes probabilidades de significación ( $\mathrm{p}$-valor) son relativamente pequeñas. No obstante, para un nivel de significación $\alpha=0,05$, el único factor que claramente influye en el nivel de sensibilización es la existencia en la empresa de trabajadores con discapacidad $(p=0,0166)$.

Las siguientes gráficas muestran los valores medios y los correspondientes intervalos de confianza al 95\% del indicador de sensibilización para los diferentes factores en estudio.

Vemos que las empresas que disponen de empleados con discapacidad presentan un mayor nivel de sensibilización, siendo la diferencia estadísticamente significativa, como se vio en la tabla ANOVA (Figura 8). 


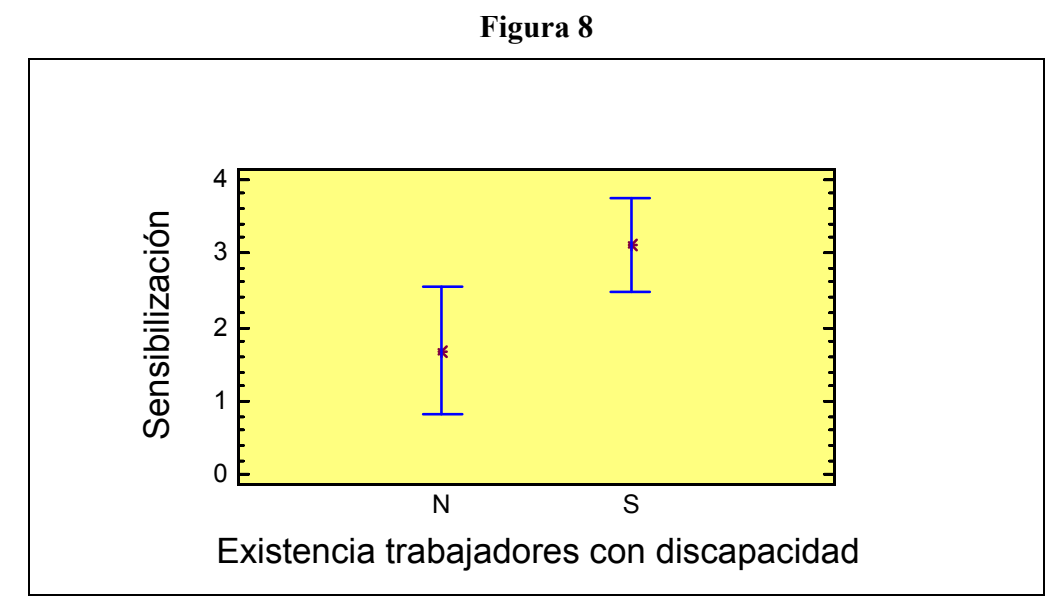

Por el tamaño de la empresa (Figura 9) se observa una mayor sensibilización en el caso de las empresas con más de 50 trabajadores, aunque no es estadísticamente significativa.

Figura 9

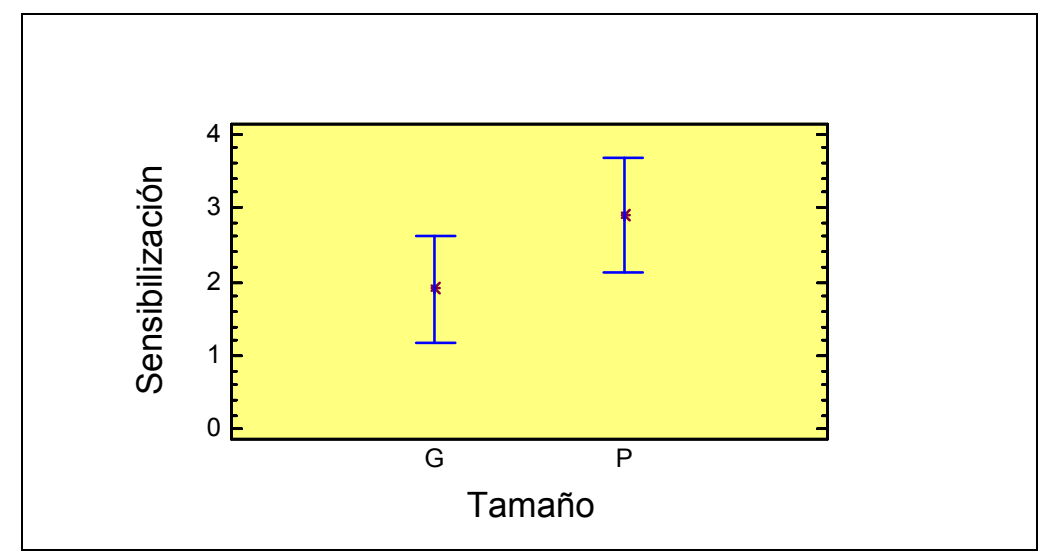

Por sectores, el sector más sensibilizado es la Administración Pública, seguido del sector Industria, siendo el sector Servicios es de menor sensibilización (Figura 10). En cualquier caso, las diferencias no son estadísticamente significativas. 
Figura 10

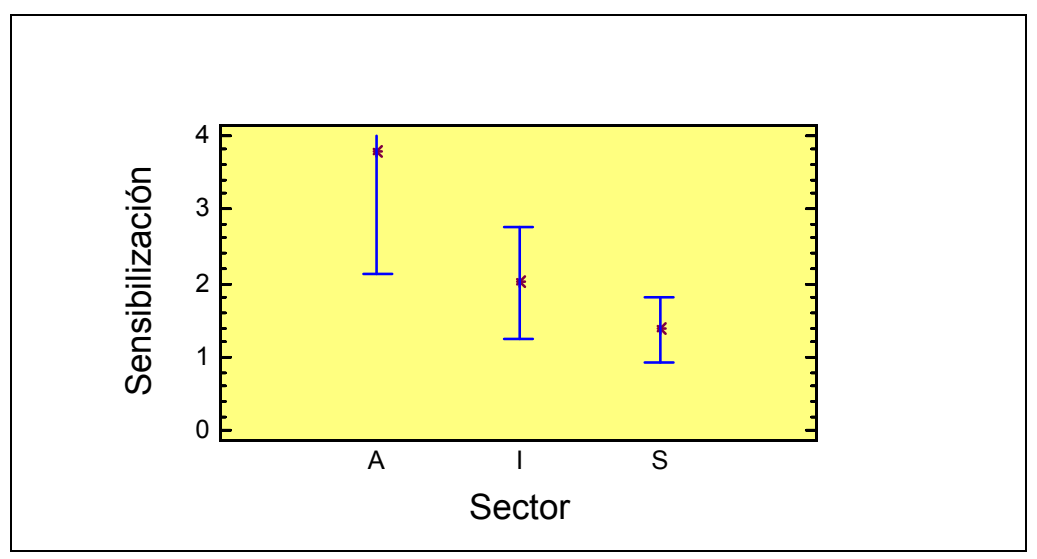

En un siguiente paso vamos a eliminar los datos correspondientes a los tres casos de administración pública. El pequeño número de observaciones impide realizar un análisis más completo que contemple las posibles interacciones de los factores.

Limitando, por tanto, el número de casos a 57 , se obtiene la siguiente tabla del Análisis de la Varianza:

Tabla 5

\begin{tabular}{|l|l|l|l|l|l|}
\hline Fuente & $\begin{array}{c}\text { Suma de } \\
\text { cuadrados }\end{array}$ & $\begin{array}{c}\text { Grados } \\
\text { libertad }\end{array}$ & $\begin{array}{c}\text { Cuadrado } \\
\text { Medio }\end{array}$ & Cociente F & p-valor \\
\hline Efectos principales & & & & & \\
\hline A) Sector & 19,8773 & 1 & 19,8773 & 5,40 & 0,0243 \\
\hline B) Tamaño & 0,145586 & 1 & 0,145586 & 0,04 & 0,8432 \\
\hline C) Trab. con discapacidad & 0,058602 & 1 & 0,058602 & 0,02 & 0,9001 \\
\hline Interacciones & & & & & \\
\hline AB & 4,14667 & 1 & 4,14667 & 1,13 & 0,2937 \\
\hline AC & 21,4412 & 1 & 21,4412 & 5,82 & 0,0195 \\
\hline BC & 1,90238 & 1 & 1,90238 & 0,52 & 0,4756 \\
\hline RESIDUOS & 184,101 & 50 & 3,68203 & & \\
\hline TOTAL (Corregido) & 240,877 & 56 & & & \\
\hline
\end{tabular}

Al tener en consideración el efecto de las interacciones, se aprecian nuevos aspectos en el análisis. Ahora vemos que el único efecto principal que claramente influye en la variable respuesta es el sector (Servicios o Industria), con una probabilidad de significación $p=0,0243$. No 
influye ni el tamaño de la empresa ni el hecho de tener o no trabajadores con discapacidad.

Las siguientes gráficas muestran los valores medios y los correspondientes intervalos de confianza al 95\% del indicador de sensibilización para los diferentes factores en estudio.

Figura 11

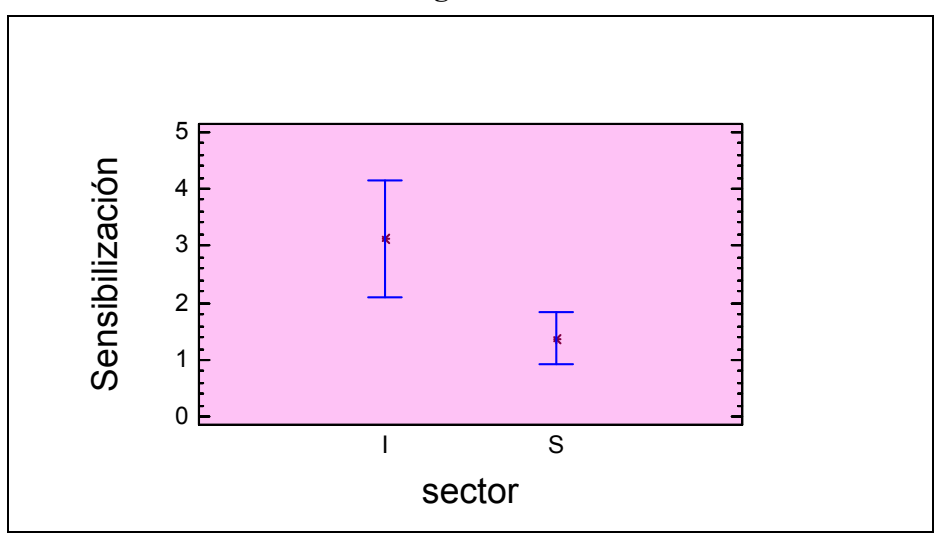

Vemos claramente (Figura 11) que las empresas pertenecientes al sector Industria presentan un valor promedio del indicador de sensibilización mayor que en el caso de las empresas pertenecientes al sector Servicios, siendo la diferencia estadísticamente significativa.

Por otra parte, observamos (Figura 12) que el tamaño de la empresa no influye en el nivel de sensibilización, presentando unos valores promedios parecidos.

Figura 12

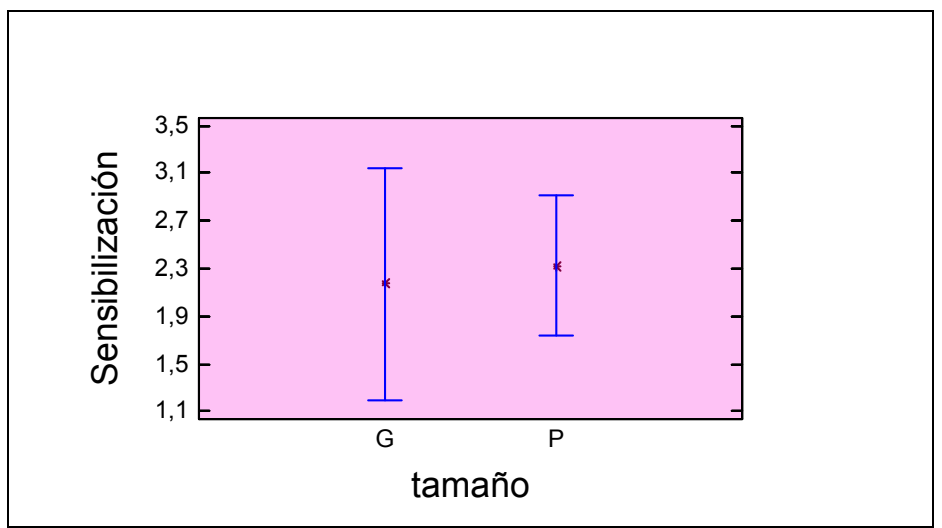


Asimismo (Figura 13), la existencia de trabajadores con discapacidad es un factor carente de influencia por sí mismo.

Figura 13

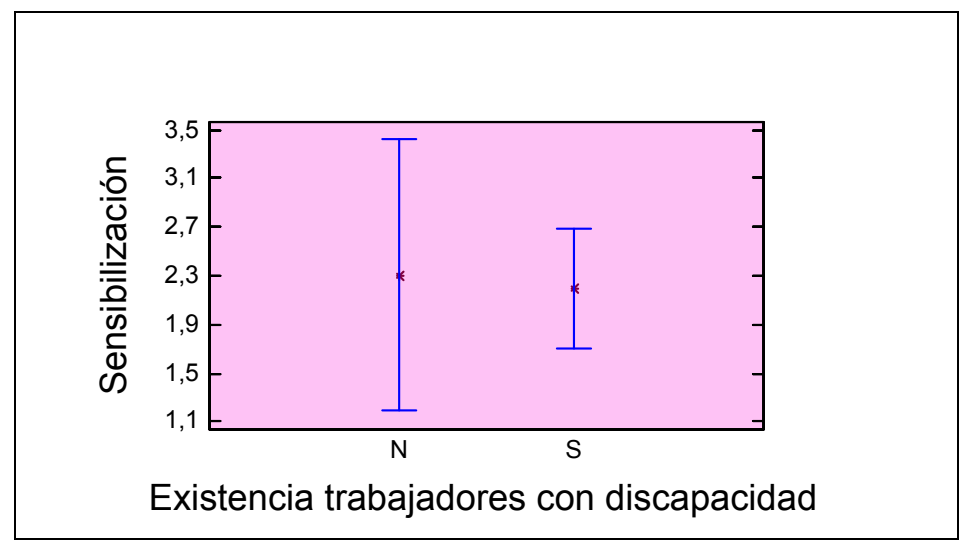

Volvamos de nuevo a la tabla ANOVA y consideremos ahora las interacciones de los factores 2 a 2 . Podemos observar que la interacción $\mathrm{AC}$, es decir la interacción del sector de la empresa con la existencia de trabajadores con discapacidad sí es un factor que influye significativamente en el indicador de sensibilización $(p=0,0195)$. Es decir, si bien como factor aislado el hecho de que la empresa tenga empleados con discapacidad no afecta al nivel de sensibilización ante el problema del empleo en este colectivo, la interacción con el sector de la empresa sí es influyente.

Vemos en el gráfico de interacción (Figura 14) que la sensibilización en el sector industrial disminuye significativamente cuando la empresa cuenta con trabajadores con discapacidad. Llama la atención porque, en principio, parecería que debería ocurrir lo contrario. La interpretación que podría darse a esta circunstancia no es nada halagüeña: parece que la experiencia en el sector Industria con trabajadores con discapacidad no es positiva. En sentido contrario, las empresas del sector Servicios son, a priori, menos receptivas o sensibilizadas ante la posibilidad de emplear a trabajadores con discapacidad. Sin embargo, cuando se consideran empresas de servicios que disponen de trabajadores con discapacidad, la percepción o sensibilidad ante el problema mejora significativamente. 

empleo de trabajadores con discapacidad en la provincia de Cádiz

Figura 14

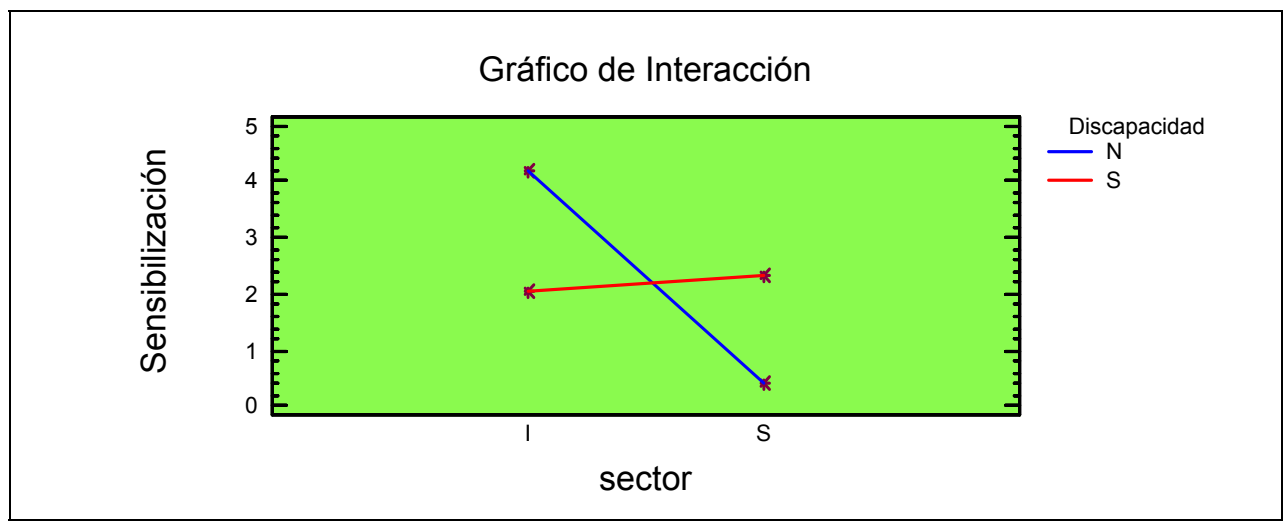

En relación con las otras dos interacciones, podemos ver en los gráficos siguientes (Figuras 15 y 16) la escasa significación de la influencia de la interacción en la variable respuesta, como ya se indicó.

Figura 15

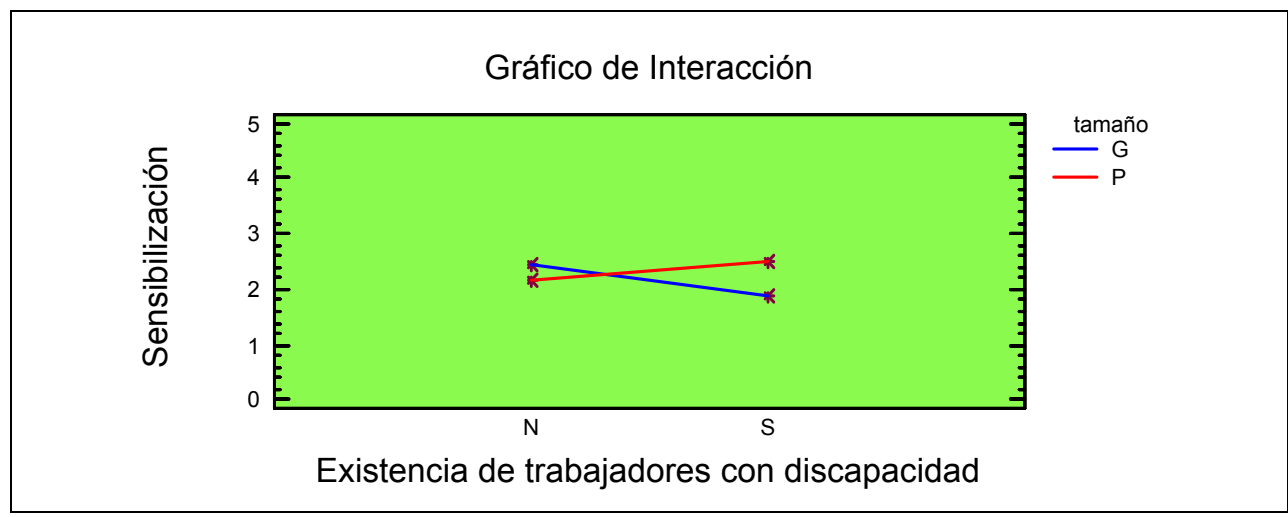

Figura 16

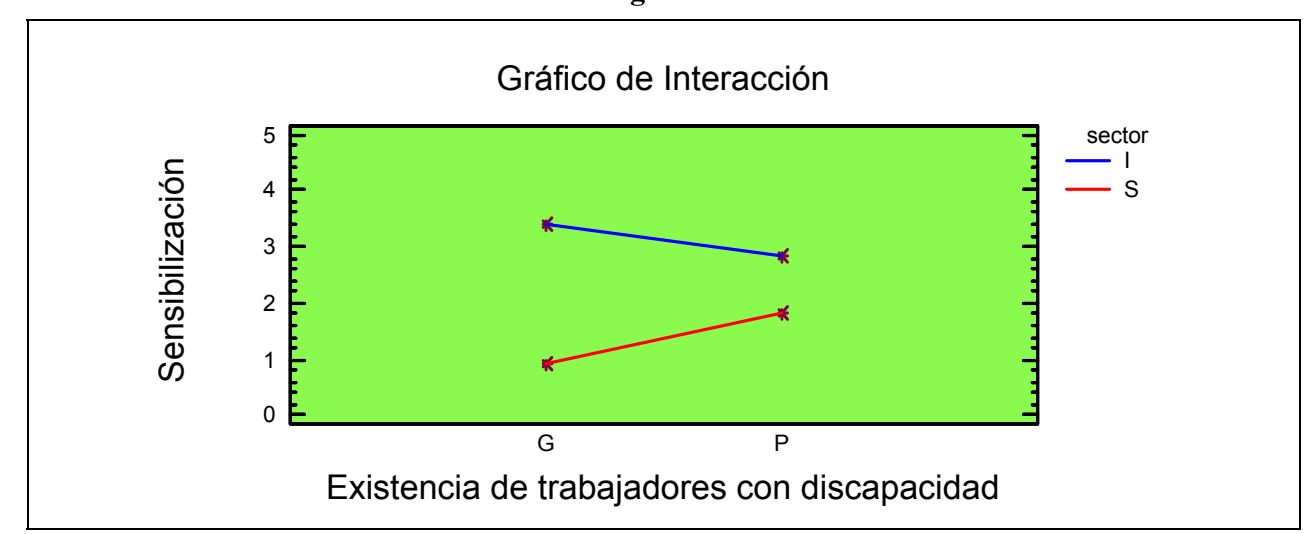




\section{BIBLIOGRAFÍA}

LeAL, Antonio y Héctor M. RAmOS (2006) Oportunidades en el empleo para personas con discapacidad física y sensorial en la provincia de Cádiz. Universidad de Cádiz: Technical Report.

MONTGOMERY, Douglas C. (2009) Design and Analysis of Experiments. Hoboken, U.K.: John Wiley \& Sons.

PEÑA, Daniel (2010) Regresión y diseño de experimentos. Madrid: Alianza Editorial. 